\title{
Ultra-High-Field MR Imaging in Polymicrogyria and Epilepsy
}

A. De Ciantis, A.J. Barkovich, M. Cosottini, C. Barba, D. Montanaro, M. Costagli, M. Tosetti, L. Biagi, W.B. Dobyns, and R. Guerrini O- $\equiv$

\section{ABSTRACT}

BACKGROUND AND PURPOSE: Polymicrogyria is a malformation of cortical development that is often identified in children with epilepsy or delayed development. We investigated in vivo the potential of $7 \mathrm{~T}$ imaging in characterizing polymicrogyria to determine whether additional features could be identified.

MATERIALS AND METHODS: Ten adult patients with polymicrogyria previously diagnosed by using 3T MR imaging underwent additional imaging at 7T. We assessed polymicrogyria according to topographic pattern, extent, symmetry, and morphology. Additional imaging sequences at 7T included 3D T2* susceptibility-weighted angiography and 2D tissue border enhancement FSE inversion recovery. Minimum intensity projections were used to assess the potential of the susceptibility-weighted angiography sequence for depiction of cerebral veins.

RESULTS: At 7T, we observed perisylvian polymicrogyria that was bilateral in 6 patients, unilateral in 3, and diffuse in 1. Four of the 6 bilateral abnormalities had been considered unilateral at 3T. While 3T imaging revealed 2 morphologic categories (coarse, delicate), 7T susceptibility-weighted angiography images disclosed a uniform ribbonlike pattern. Susceptibility-weighted angiography revealed numerous dilated superficial veins in all polymicrogyric areas. Tissue border enhancement imaging depicted a hypointense line corresponding to the gray-white interface, providing a high definition of the borders and, thereby, improving detection of the polymicrogyric cortex.

CONCLUSIONS: 7T imaging reveals more anatomic details of polymicrogyria compared with 3T conventional sequences, with potential implications for diagnosis, genetic studies, and surgical treatment of associated epilepsy. Abnormalities of cortical veins may suggest a role for vascular dysgenesis in pathogenesis.

ABBREVIATIONS: FSPGR = fast-spoiled gradient echo; GRE = gradient-recalled echo; IR = inversion recovery; SWAN = susceptibility-weighted angiography; $\mathrm{TBE}=$ tissue border enhancement

$\mathbf{P}$ olymicrogyria is a malformation of the cerebral cortex secondary to abnormal migration and postmigrational development. ${ }^{1}$ It is characterized by an excessive number of abnormally small gyri separated by shallow sulci, associated with fusion of the

Received June 9, 2014; accepted after revision August 1.

From the Pediatric Neurology Unit (A.D.C., C.B., R.G.), Meyer Children's Hospital, University of Florence, Florence, Italy; Department of Radiology and Biomedical Imaging (A.J.B.), University of California San Francisco, San Francisco, California; Department of Translational Research and New Technologies in Medicine and Surgery (M. Cosottini), University of Pisa, Pisa, Italy; IMAGO7 Foundation (M. Cosottini), Pisa, Italy; Fondazione Consiglio Nazionale delle Ricerche/Regione Toscana (D.M.), Unità Operativa Semplice Neuroradiologia, Pisa, Italy; Istituto di Ricovero e Cura a Carattere Scientifico Stella Maris Foundation (M. Costagli, M.T., L.B., R.G.), Pisa, Italy; and Center for Integrative Brain Research (W.B.D.), Seattle Children's Hospital, Seattle, Washington.

This work was supported by the Pisa Foundation (Project 133/11 "Ultra-high field MRI targeted imaging of dysplastic cortical lesions and dysembryoplastic tumours"), the European Research Project on Rare Diseases (E-Rare-2, TUBGENCODEV, 11-027), and the European Union Seventh Framework Programme FP7/2007-2013 (under the project DESIRE: Development and Epilepsy: Strategies for Innovative Research to improve diagnosis, prevention and treatment in Children with difficult to treat Epilepsy, Grant Agreement: 602531). overlying molecular layer (layer 1 ) of the cerebral cortex. ${ }^{2}$ This combination of features produces a characteristic appearance of irregularity at both the cortical surface and cortical-white matter junction. ${ }^{3,4}$ Its pathogenesis is still poorly understood, and its histopathology, clinical features, topographic distribution, and imaging appearance are heterogeneous. Deficiencies in the understanding of this malformation result from both causal heterogeneity (causative factors include destructive events such as congenital infections, ${ }^{5,6}$ in utero ischemia, ${ }^{7}$ metabolic disorders, and

\footnotetext{
Please address correspondence to Renzo Guerrini, MD, Pediatric Neurology and Neurogenetics Unit and Laboratories, Meyer Children's Hospital, Department of Neuroscience, Pharmacology and Child Health, University of Florence, Viale Pieraccini 24, 50139, Florence, Italy; e-mail: renzo.guerrini@unifi.it

- Indicates open access to non-subscribers at www.ajnr.org

三 Indicates article with supplemental on-line tables.

Indicates article with supplemental on-line photos.

http://dx.doi.org/10.3174/ajnr.A4116
} 
several gene mutations and copy number variations ${ }^{1,8,9}$ ) and the limited number of postmortem examinations available.

The topographic distribution of polymicrogyria may be focal, multifocal, or diffuse; unilateral or bilateral; and symmetric or asymmetric. ${ }^{10-15}$ Polymicrogyria can occur as an isolated disorder or can be associated with other brain abnormalities such as corpus callosum dysgenesis, cerebellar hypoplasia, schizencephaly, and periventricular and subcortical heterotopia. ${ }^{16,17}$

Clinical manifestations of patients with polymicrogyria have a large spectrum, ranging from isolated selective impairment of cognitive function ${ }^{18}$ to severe encephalopathy and intractable epilepsy. ${ }^{19}$ The severity of neurologic manifestations and the age at presentation are, in part, influenced by the extent and location of the cortical malformation but may also depend on its specific etiology.

Neuroimaging has a primary role in the diagnosis and classification of polymicrogyria due to its noninvasive nature. Imaging findings are variable and are primarily determined by the morphology of the malformed cortex itself but also by the maturity of myelination and imaging-related technical factors (section thickness, gray-white matter contrast) ${ }^{20}$ In addition, polymicrogyria-like patterns can be seen in certain malformations, such as tubulinopathies ${ }^{21}$ and cobblestone malformations ${ }^{22-24}$; these have different histologic appearances but similar MR imaging appearances to polymicrogyria, which can lead to false diagnoses.

On the basis of morphologic characteristics, Barkovich ${ }^{2,20}$ described the variable appearance of polymicrogyria on MR imaging and suggested that the gyral-sulcal dysmorphisms may be roughly divided into 3 main categories: coarse with a thick, bumpy cortex and irregular surface on both the pial and gray-white junction sides; delicate with multiple small, fine gyri of thin cortex that remains thin even after myelination; and sawtooth, composed of thin microgyri separated by deep sulci (primarily seen in diffuse polymicrogyria and before myelination develops). However, numerous gradations of morphology exist within these groups. To date, neither functional nor etiologic associations have been inferred on the basis of this imaging categorization of a polymicrogyric cortex.

Over the past several years, ultra-high-field 7T MR imaging has been available for in vivo human brain imaging. In vivo 7T MR imaging can provide greater tissue-type identification than that obtained in vitro without stains. ${ }^{25}$ As a result of an increased signal-to-noise ratio, enhanced image contrast, and improved resolution, MR imaging at $7 \mathrm{~T}$ can visualize small anatomic structures not previously appreciated at lower fields. ${ }^{25-28}$ Because $7 \mathrm{~T}$ MR imaging has already provided diagnostic benefits in different pathologies ${ }^{28}$ such as multiple sclerosis, ${ }^{29}$ cerebrovascular diseases (strokes, microbleeds), ${ }^{30,31}$ aneurysms, ${ }^{32}$ cavernous malformations, ${ }^{33}$ brain tumors, ${ }^{34}$ and degenerative brain diseases like dementia and Parkinson disease, ${ }^{35,36}$ we tested the added value of 7T MR imaging in providing details of structural changes and their extent in 10 patients with polymicrogyria with respect to conventional $3 \mathrm{~T}$ imaging. We also addressed the limitations we encountered while exploring the polymicrogyric brain with $7 \mathrm{~T}$.

\section{MATERIALS AND METHODS Ethics Statement}

Written informed consent was obtained from all patients and is recorded on file. The experimental protocol named "Evaluation of dysplastic cortical lesions and dysembryoplastic tumors by using ultra-high field MR imaging target imaging" Project 133/11 was funded by the Pisa Foundation and approved by the local competent ethics committee and the Italian Ministry of Health. The procedures followed were in accordance with institutional guidelines and included an adverse event form.

\section{Subjects}

Between June 2013 and October 2013, we enrolled, from our cortical malformation data base, 10 adult patients (4 men and 6 women) with polymicrogyria previously imaged at $3 \mathrm{~T}$. Exclusion criteria were age younger than 18 years and any contraindications to MR imaging. Polymicrogyria, as assessed by previous 3T MR imaging, had been classified as bilateral perisylvian in 2 patients, unilateral perisylvian in 7 patients, and diffuse in 1 patient. The mean age was 30.1 years (range, 21-53 years). Clinical details are described in On-line Table 1.

\section{Data Acquisition}

All 10 patients were imaged at both 3T (Excite HDx; GE Healthcare, Milwaukee, Wisconsin, equipped with a GE 8-channel head coil) and 7T (Discovery MR 950 scanner; GE Healthcare, equipped with a 2-channel transmit/32-channel-receive head coil, Nova Medical, Wilmington, Massachusetts). All participants received earplugs and a pair of pads covering the ears to limit acoustic noise.

The 3T MR imaging standard protocol included the following sequences: 3D T1-weighted fast-spoiled gradient echo (FSPGR), 2D T2 FLAIR, 2D T2-weighted FSE, and 2D white matter-suppressed FSE inversion recovery (IR). The 7T MR imaging protocol included the following sequences: 3D T1-weighted FSPGR, 3D susceptibility-weighted angiography (SWAN), 2D T2*-weighted targeted dual-echo gradient-recalled echo (GRE), 2D T2weighted FSE, and 2D gray-white matter tissue border enhancement (TBE) FSE-IR. TBE is an IR sequence that uses an appropriate $\mathrm{TI}$ to produce images in which the interface between 2 neighboring tissues of interest is hypointense; therefore, tissue borders are clearly represented by dark lines. This effect is achieved by setting imaging parameters so that the neighboring tissues have magnetization with equal magnitude but out of phase; therefore, the voxels containing a mixture of each tissue (ie, the tissue interface) possess minimal net signal. ${ }^{37}$ Therefore, the hypointense line-marking tissue interface produced by TBE does not reflect any specific fiber structure. Details of imaging parameters for 3T and 7T imaging are shown in On-line Table 2.

To limit specific absorption rate-related problems, image distortions and stronger susceptibility phenomena related to ultrahigh-field, whole-brain 7T imaging included only SWAN and FSPGR sequences, with the remaining sequences targeting specific regions of interest by using small FOVs and a limited number of sections. To select the areas of interest for targeted imaging, we were guided by initial 7T whole-brain sequences. 


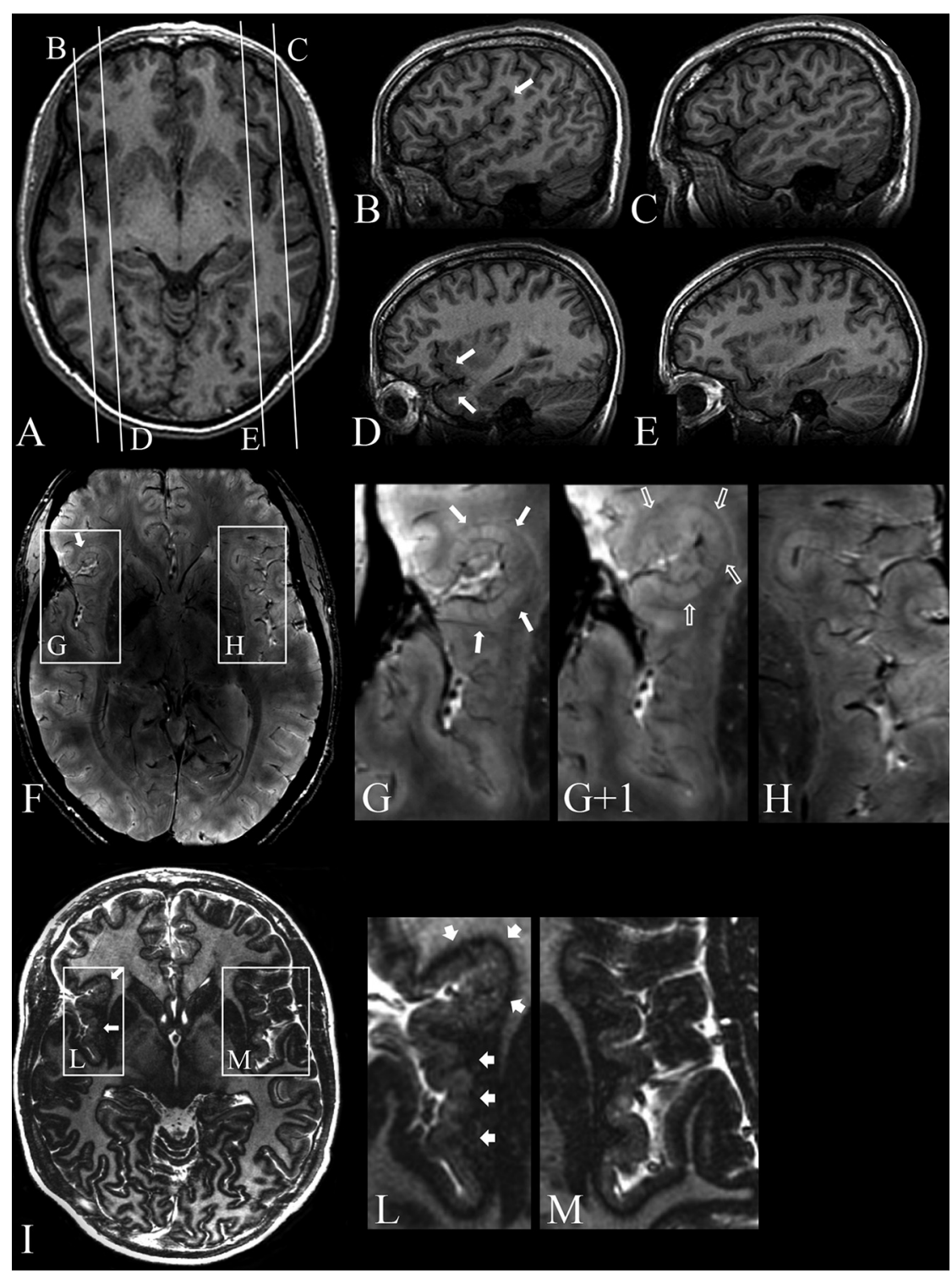

FIG 1. Patient 8. 3T axial $(A)$ and sagittal (B-E) 3D FSPGR, 7T 3D SWAN $(F)$, and magnified images (G, $G+1, H), 7$ T axial 2D TBE FSE-IR (I) and magnified images ( $L$ and $M)$. $A$, Mild cortical thickening in the right frontal operculum. Contiguous sagittal sections across the frontal operculum and the Sylvian fissure on the right $(B$ and $D)$ and left $(C$ and $E$ ) sides provide a better comparative view of the morphologic characteristics of malformed-versus-normal cortex. B, An abnormal right Sylvian fissure (arrow), which is vertically oriented, shortened, and bordered by thick and irregular cortex. D, Thickening of the cortex in the inferior frontal gyrus and superior temporal gyrus (arrows). F, Two contiguous expanded views, from caudal $(G)$ to rostral $(G+7)$, provide ultra-high-resolution details of the right frontal operculum, which are not visible at $3 T(A-E)$, substantiating the presence of a polymicrogyric cortex. $\mathrm{H}$, A magnification of the homologous contralateral region clearly enhances the appreciation of the difference in folding of the polymicrogyric and normal cortex. I, Magnifications $(L$ and $M$ ) show a hypointense line representing the gray-white matter interface and provide a high definition of the polymicrogyric (L, arrows) and normal $(M)$ cortex, making it easier to appreciate irregularities in thickness and folding of the polymicrogyric cortex.

\section{Data Analysis}

All 7T images were independently assessed by 3 reviewers and later as a group to reach a consensus for eligibility. 3T and 7T images were assessed separately in each patient. Polymicrogyria was diagnosed with satisfaction of the combination of these characteristics: 1) the cortex had an irregular outer surface with an abnormal gyral pattern, 2) the cortex appeared thickened or overfolded, and 3) the cortical-white matter junction was irregular. ${ }^{38}$

We reviewed all images for the following findings: 1) topo- graphic pattern of polymicrogyria, 2) extent, 3) symmetry (if bilateral), 4) range of gyral-sulcal dysmorphisms, and 5) associated abnormalities. We classified the topographic pattern according to the different patterns described in the literature: focal, bilateral frontal, bilateral perisylvian (which varies in the extent of involvement), bilateral parieto-occipital, and diffuse polymicrogyria.

The 3T and 7T images were assessed digitally with a workstation (Advantage 4.1; GE Healthcare). 3D images were reformatted in orthogonal planes to better evaluate the cortical thickening and to rule out partial volume artifacts.

The postprocessing of SWAN images was performed by using the minimum intensity projection algorithm and multiplanar reformation techniques. The minimum intensity projection algorithm has the characteristic of enhancing the visualization of veins while attenuating the signal from the brain tissue. Conversely, tissue border enhancement images do not require additional postprocessing.

\section{RESULTS}

All imaging sessions were well-tolerated by the patients, and no adverse events occurred. Brain MR imaging findings and clinical details of all patients are summarized in On-line Table 1.

At 3T, we observed perisylvian polymicrogyria that was unilateral in 7 patients and bilateral in 2. Of the 7 patients with unilateral polymicrogyria, 6 had multilobar involvement, and in 1 , only a portion of the Sylvian fissure was involved (Fig 1). One patient had diffuse polymicrogyria.

A range of gyral-sulcal dysmorphisms emerged at 3T (Fig 2A and On-line Fig $1 A$ ), all falling into the categorization formulated by Barkovich, ${ }^{2}$ with 6 of 10 patients having dysmorphisms with a coarse appearance and the remaining 4 having a coarse together with a delicate appearance.

The thickness of the polymicrogyric cortex, measured with FSPGR imaging, ranged from 3.7 to $12 \mathrm{~mm}$ (the thickness of the normal cortex at $3 \mathrm{~T}$ ranged from 1.6 to $4 \mathrm{~mm}$ ), with great variability even between adjacent cortical regions.

In 3 patients, polymicrogyria was an isolated abnormality, while in the remaining 7 patients, we detected additional subcortical abnormalities: unilaterally $(n=1)$ or bilaterally $(n=4)$ dilated ventricles, unilateral $(n=2)$ or bilateral $(n=2)$ hippocampal abnormalities, absence of the septum pellucidum $(n=$ $2)$, cavum vergae $(n=1)$, unilateral periventricular nodular het- 
erotopia $(n=2)$, and unilateral open-lip schizencephaly $(n=1)$. White matter volume reduction was apparent under the polymicrogyric cortex in all patients. One patient exhibited left intraor-

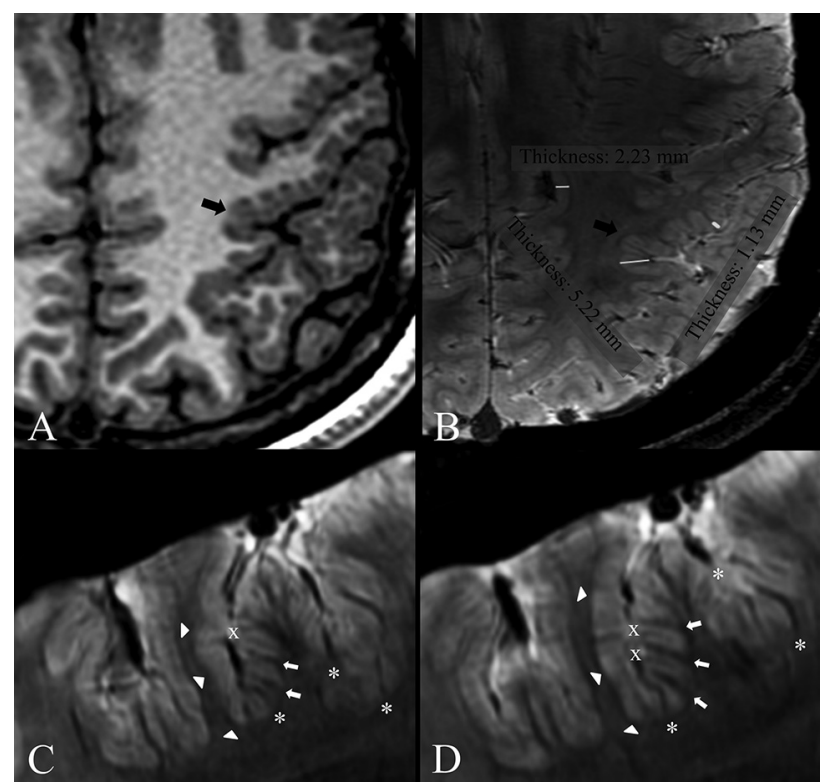

FIG 2. Patients $1(A$ and $B)$ and $9(C$ and $D)$. Comparison of $3 T$ axial 3D FSPGR $(A)$ and 7T axial 3D SWAN (B) images. Contiguous 7T axial 3D SWAN acquisitions show anatomic details of the polymicrogyric cortex $(C$ and $D)$. A, Delicate appearance of the polymicrogyric cortex in the left pre- and postcentral sulci, characterized by multiple small and delicate gyri of thin cortex (arrow). B, A thin and undulated polymicrogyric cortex, in which the spaces between microgyri are filled by thin white matter digitations, which have a low periodicity and are loosely packed. The gray-white matter junction is bordered by a thin hypointense line. 7T can, therefore, resolve the individual microgyri, revealing how grossly different morphologic characters (coarse or delicate) at $3 T$ result, in fact, from variations of a common underlying morphologic pattern. $B$, Examples of cortical thickness measurements of normal $(2.23 \mathrm{~mm})$ and polymicrogyric cortex $(1.13-5.22 \mathrm{~mm})$ by using the straight-line distance measure between the surface and depth of the cortex. $C$ and $D$, SWAN images show details of cortical structures and allow disentanglement of the structural units underlying the radiologic appearance of polymicrogyria. The typical undulated aspect is clearly detectable following the hypointense lines of the cortical border (arrowheads), which we assume represent arcuate white matter fibers, the white matter digitations within the gyri (arrows), the small vessels joining the pial veins (asterisks), and the fused molecular layer (crosses).
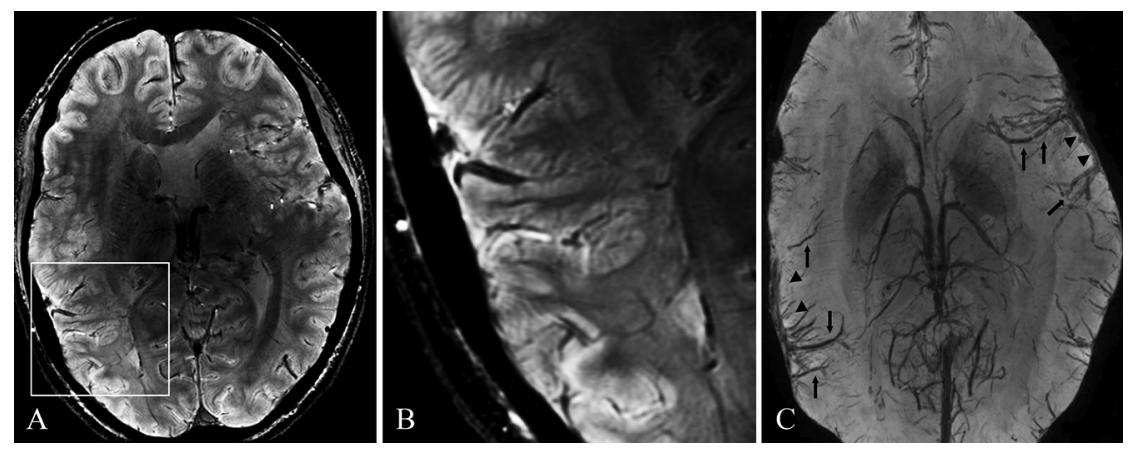

FIG 3. Patient 9. 7T axial 3D SWAN (A, magnified in $B$ ) and its minimum intensity projection reconstruction $(C)$. A, Bilateral polymicrogyria involving the left frontal operculum and Sylvian fissure and the right Sylvian fissure and temporal lobe. B, Details of the polymicrogyric cortex in the right temporal lobe (magnified). $C$, Minimum intensity projection reconstruction shows dilated superficial veins in correspondence with the polymicrogyric areas (arrowheads); the large vascular structures running through the polymicrogyric sulci (arrows) define the location and the extent of the malformation. bital cavernous hemangiomas and a left soft-tissue hemangioma and hypertrophy of the right Sylvian and medullary veins.

7T provided additional details to $3 \mathrm{~T}$ findings and revealed more extensive areas of polymicrogyria in all patients. In particular, in 3 patients (patients 4, 7, and 8) in whom 3T FSPGR imaging had revealed unilateral polymicrogyria, 7T SWAN imaging confirmed a unilateral distribution but detected more extensive involvement (Fig 1). In 3 patients with bilateral polymicrogyria at 3T (patients 1, 3, and 9), 7T SWAN revealed more extensive involvement. Four patients (patients 2, 5, 6, and 10) who had been classified as having unilateral polymicrogyria at $3 \mathrm{~T}$ exhibited bilateral involvement at 7T (On-line Fig 2). In patient 2, 3T FSPGR showed left posterior polymicrogyria engaging the left temporoinsuloparietal region and the medium and superior occipital gyri. 7T SWAN imaging disclosed that polymicrogyria also involved the right posterior insula. In patient 5 , in whom 3T FSPGR showed left frontotemporoparietal polymicrogyria along the whole Sylvian fissure, 7T SWAN revealed polymicrogyria also in the right frontal operculum. In patients 6 and 10, the polymicrogyric cortex had right temporoparietal distribution at $3 \mathrm{~T}$ FSPGR, while 7T disclosed abnormal infolding and thickening of the left frontal operculum. In patient 10, 7T SWAN also showed that polymicrogyria involved the area around the whole left Sylvian fissure, left anterior insula, and left anterior temporal region.

The increased signal-to-noise ratio and the increased sensitivity to magnetic susceptibility effects on SWAN sequences at $7 \mathrm{~T}$ combined with high-resolution images with enhanced intracortical contrast provided improved polymicrogyria detailing by making it possible to identify details in the entire cortex: the lowest part of the sulcus, the intermediate zone, and the crown of the gyrus. The different categories of gyral and sulcal dysmorphisms seen at 3T (coarse, delicate, sawtooth) were not identifiable as such at $7 \mathrm{~T}$, in that SWAN images revealed a homogeneous morphologic character (Fig $2 B$ and Online Fig $1 B$ ). The malformed cortex, measured by using SWAN imaging, was 0.78 - to $7-\mathrm{mm}$ thick (the normal cortex was $1-2.89 \mathrm{~mm}$ ), exhibiting an undulated profile at the gray-white matter junction and bordered by a thin hypointense line, which was considered to represent the arcuate white matter fibers. Within the abnormal cortex, small linear hypointense transcortical lines perpendicular to the cortical layers, probably representing the larger transcortical blood vessels, were present. The hypointense lines of the cortical border combined with those within the cortex contributed to generating the typical polymicrogyric appearance of the cortical ribbon at 7T (Fig 2C, $-D$ ).

In all patients, SWAN imaging revealed numerous dilated superficial veins draining the deeper infoldings of polymicrogyria, which were not visible at $3 \mathrm{~T}$ by using conventional (FSPGR, FSE, and IR) sequences. These large venous structures, which have been described previously, ${ }^{39}$ appeared to be roughly proportional to 
the depth/size of the cortical infolding and extent of polymicrogyria (Fig 3).

In all patients, TBE imaging revealed a hypointense line corresponding to the gray-white matter interface, providing a high definition of the borders of the polymicrogyric cortex (On-line Figs 3 and 4). TBE imaging substantiated SWAN findings and, providing a precise topographic definition of the gray-white matter junction, made the examiner more confident about the extent of polymicrogyria, especially when the border between gray and white matter was poorly defined or the polymicrogyric cortex merged with a seemingly normal cortex (On-line Figs 3 and 4).

The diagnostic gain obtained at $7 \mathrm{~T}$ originated from the improved image and contrast resolution of SWAN sequences and, to a lesser degree, from 2D GRE images. GRE sequences revealed cortical and pial veins and small vessels inside the cortex less precisely than SWAN. With GRE, cortical layering was less defined, the arcuate white matter fibers could not be visualized, and white matter digitations within the gyri were less conspicuous than those observed by using SWAN or TBE. GRE defined the complexity of cortical gyration better than FSPGR but worse than SWAN and TBE. T2-weighted FSE at 7T did not provide additional information with respect to the same sequence at $3 \mathrm{~T}$ and had lower contrast and spatial resolution than GRE.

\section{DISCUSSION}

Accurate MR imaging depiction of polymicrogyria has important implications for correlating clinical severity with the extent of cortical abnormality, ${ }^{20,38,40}$ guiding genotyping of specific malformation patterns, ${ }^{1}$ and delineating the resection margins in patients who are candidates for epilepsy surgery. ${ }^{41-44}$

In this series, 7T SWAN imaging provided improved structural cortical details with more accurate information on cortical thickness, sulcal pattern, and cerebral veins. At 7T, the polymicrogyric cortex exhibited a highly variable thickness, ranging from 0.78 to $7 \mathrm{~mm}$, close to pathology-specimen measures, ${ }^{25}$ with bumpy inner and outer surfaces, broad gyri, and shallow sulci.

7T SWAN imaging also allowed better qualitative assessment of sulcal patterns compared with conventional $3 \mathrm{~T}$ imaging. Due to high spatial resolution and increased contrast within the cortex of 7T SWAN imaging, the gyral-sulcal dysmorphisms of polymicrogyria $^{2}$ visible at 3 T FSPGR appeared as a highly characteristic undulated pattern of contiguous densely packed microgyri. The polymicrogyric cortex, seen in some of the 3T FSPGR images as thick, rough, and blurred, was merely a poorly resolved image of more deeply undulating thin gyri, with the apparent cortical thickening generated by lower contrast and resolution that result from conventional sequences and field strengths. The significance of the deeper undulations remains to be determined.

Combining $7 \mathrm{~T}$ high spatial resolution with 3D SWAN sequences. we uncovered bilateral involvement in 4 patients who had been diagnosed as exhibiting unilateral abnormalities at 3T. In addition to the SWAN imaging, we used a custom-designed TBE sequence, which produced a hypointense line along the graywhite matter interface, thereby providing a high definition of the undulating polymicrogyric borders and, together with improved resolution on SWAN images, allowed improved differentiation from adjacent normal cortex. TBE images substantiated the extent of malformation observed by using SWAN imaging and revealed the borders of polymicrogyria in those areas where the gray-white matter junction was poorly defined or the polymicrogyric cortex merged with the normal cortex.

In addition to producing more accurate morphologic details of the polymicrogyric cortex, SWAN imaging at 7T detected deoxygenated venous blood that allowed visualization of the superficial and deep cerebral veins. Although 3T SWAN can also visualize venous abnormalities, there is no previous report emphasizing this finding at 3T, to our knowledge. At 7T, the polymicrogyric cortex was shown to harbor numerous dilated superficial veins, whose representation seemed to be roughly proportional to the extent and severity of the cortical malformation. The pial veins were visible as linear hypointensities, orthogonal to and penetrating into the cortex. Following the expected course of the sulci, the pial veins defined the complexity of cortical gyration, revealing the contour of polymicrogyria. SWAN-minimum intensity projection images, which enhance the visualization of veins while attenuating the signal from brain tissue, revealed large vascular structures running through the sulci, draining from the polymicrogyric cortex into the pial veins. These findings reinforce previous imaging observations indicating anomalous venous drainage in dysplastic cortical areas. ${ }^{39,45}$ On the other hand, although perfusion failure is a recognized cause of polymicrogyria, there is no reported neuropathologic evidence of vascular malformations associated with it. ${ }^{6,46-49}$

Abnormal venous drainage might develop as a response to an event, such as cortical injury, during fetal development ${ }^{50}$ and is probably the result of a lack of condensation of cortical veins with persistence of embryonic dural plexus tributaries. ${ }^{45}$ Based on the prominence of vascular abnormalities in polymicrogyria, one may hypothesize that they are involved in its etiopathogenesis. For instance, a causative role of venous abnormalities has been suggested in patients with Sturge-Weber syndrome and polymicrogyria in the region underlying pial angiomatosis. ${ }^{38,51,52}$ One hypothesis concerning the cause of Sturge-Weber is that leptomeningeal vascular dysplasia leads to impaired perfusion, especially in regard to microvenular drainage, which produces blood stasis with progressive hypoperfusion and ischemia. ${ }^{53}$ Alternatively, cortical dysgenesis could result from the abnormal expression of a factor playing a role in both vascular and cortical development. ${ }^{54}$ On the basis of the observation that cryogenic lesions in the cortex of neonatal rats can produce focal microgyria and subsequent abnormal vascularization, it has also been suggested that cortical venous abnormalities in polymicrogyria might be the consequence, and not the cause, of abnormal cortical development. ${ }^{55}$ However, it is not clear that lesion-induced polymicrogyria in the normally agyric rat cortex is the same malformation as the polymicrogyria found in gyrencephalic humans.

On the basis of the frequent location of polymicrogyria in watershed vascular territories and on evidence of laminar necrosis in brain specimens, ${ }^{7,49,56-59}$ some authors have suggested that polymicrogyria might result from arterial ischemia during the second trimester of gestation. Due to lack of arterial sequences in our study, however, we could not exclude the presence of arterial dysplasia in our patients and can only speculate on arterial dysgenesis as a possible cause of prenatal ischemia. In addition, we 
studied adult patients with normal or nearly normal cognitive function whose main clinical problem was epilepsy. These clinical features might have characterized a subpopulation with a specific subtype of polymicrogyria or a specific etiology, which is certainly not representative of the larger population of individuals with polymicrogyria, in which varied etiologic factors other than vascular might be prevalent.

Polymicrogyria and polymicrogyria-like malformations have indeed been associated with mutations of various genes, including COL18A1, PAX6, KIAA1279, RAB3GAP1, RAB3GAP2, TUBA1A, TUBB2B, TUBB3, TUBA8, TUBB5, TBR2, and WDR62 and different copy number variations. ${ }^{8,60-64}$ However, paucity of neuropathologic observations limits our ability to understand how comparable morphologic changes are in these conditions. Moreover, the large majority of cases of polymicrogyria are not caused by alterations of any known gene. 7T MR imaging will hopefully make it possible to better evaluate and classify specific polymicrogyria phenotypes and, by reducing sample heterogeneity, improve the approach to genetic studies, leading to a higher diagnostic yield. Genetic characterization of patients with polymicrogyria might ultimately lead to improved outcome prediction. ${ }^{65}$ Quantitative measurements of abnormal sulcal patterns have made it possible to correlate morphologic abnormalities with language impairment. ${ }^{66}$ Future $7 \mathrm{~T}$ functional MR imaging studies are needed to clarify the correlation between abnormal sulcal patterns and functional impairment.

Approximately $78 \%-87 \%$ of patients with polymicrogyria have epilepsy. ${ }^{38,67}$ Associated epilepsy is often intractable, but only a limited number of patients are eligible for surgical treatment and invasive recordings are necessary in most to define eloquent cortex and the area to be removed. ${ }^{41,43,44}$ Although the final surgical margins are determined by the use of depth electrode recordings or by corticography in the operating theater, 7T MR imaging may help presurgical planning for patients with polymicrogyria-related epilepsy by providing a clearer assessment of the margins of the polymicrogyric cortex and revealing previously undetected bilateral abnormalities.

Although we demonstrated some obvious advantages offered by 7T, we also found limitations inherent its use. The protocols and acquisition methods we used for 3T and 7T MR imaging were different, and both relaxivity and susceptibility effects differ significantly at these various field strengths. In addition, optimal protocols at 7T have not yet been implemented. SWAN and TBE sequences were not included in our standard 3T MR imaging protocol and could not be directly compared with 7T acquisitions. When imaged at conventional field strengths, patients with polymicrogyria are not evaluated with GRE images but primarily with FSPGR, FSE, or IR sequences. At 7T, the inhomogeneous radiofrequency energy deposition and the increased susceptibility effect may cause signal inhomogeneity or image distortion. To remain within the specific absorption rate limits and reduce image distortion and stronger susceptibility phenomena, we acquired targeted images with small FOVs and a few sections focused on regions of interest at 7T. Therefore, we could not image the entire brain as instead it was feasible at lower field strengths. In particular, in order not to exceed the specific absorption rate limits imposed by regulations (International Electrotechnical Com- mission Standards for Medical Electrical Equipment 60601-233), for FSE, it was possible to acquire 10 sections within 1 single acquisition, while for TBE, 10 sections were acquired during 2 separate series of 5 sections each.

An additional limitation of our study is that none of our patients had undergone epilepsy surgery and no pathology and postoperative outcome were available to be correlated with 7T imaging. Furthermore, our MR imaging comparison was based on visual, qualitative analysis. Nevertheless, we found substantially more and different information at 7T that we hope will be useful in future analyses of patients with polymicrogyria.

\section{CONCLUSIONS}

Ultra-high-field 7T imaging allows assessment of structural brain abnormalities that cannot be obtained from conventional MR imaging and represents an important tool for the diagnosis and characterization of polymicrogyria.

Disclosures: Alessio De Ciantis—RELATED: Grant: Pisa Foundation, Pisa, Italy (Project 133/11), ${ }^{*}$ Comments: My salary was paid by Pisa Foundation through Meyer Children's Hospital; Carmen Barba—RELATED: Grant: Fondazione Cassa di Risparmio Pisa, Pisa, Italy (Project 133/11)*; European Union Seventh Framework Programme FP7/20072013 under the project DESIRE (Development and Epilepsy: Strategies for Innovative Research to improve diagnosis, prevention and treatment in Children with difficult to treat Epilepsy - Grant Agreement: 602531), ${ }^{*}$ Comments: Grants allowed allocating human resources needed to perform the work for this article and comply with bureaucratic requirements; Support for Travel to Meetings for the Study or Other Purposes: Fondazione Cassa di Risparmio Pisa, Italy (Project 133/11)*; European Union Seventh Framework Programme FP7/2007-2013 under the project DESIRE (grant agreement No. 602531), ${ }^{\star}$ Comments: The reimbursement of travel expenses was made through my Institution. Mauro Costagli-RELATED: Grant: Fondazione Pisa, ${ }^{*}$ Comments: My institution received a grant from "Fondazione Pisa" for this study. Michela Tosetti—RELATED: Grant: Pisa Foundation, Pisa, Italy, ${ }^{*}$ Comments: My Institution, IRCCS Stella Maris Foundation, received a grant from "Fondazione Pisa" for a research project (Project 133/11) relating to the subject of this work. Laura BiagiRELATED: Pisa Foundation, Pisa, Italy (Project 133/11),, Comments: My institution received a grant from "Fondazione Pisa" for a research project relating to the subject of this work. William B. Dobyns-RELATED: Grant: National Institutes of Health,* Comments: grant No. R01NS058721; Grants/Grants Pending: National Institutes of Health*; Payment for Lectures (including service on Speakers Bureaus): Novartis Neuroscience Institute (Boston) in 2013, Comments: total less than US $\$ 3000$. Renzo Guerrini-RELATED: Grant: European Union Seventh Framework Programme FP7/ 2007-2013 under the project DESIRE (grant agreement No. 602531)*; Project 133/11" Fondazione Pisa. * *Money paid to the institution.

\section{REFERENCES}

1. Barkovich AJ, Guerrini R, Kuzniecky RI, et al. A developmental and genetic classification for malformations of cortical development: update 2012. Brain 2012;135:1348-69

2. Barkovich AJ. MRI analysis of sulcation morphology in polymicrogyria. Epilepsia 2010;51:17-22

3. Barkovich AJ, Kuzniecky RI, Jackson GD, et al. A developmental and genetic classification for malformations of cortical development. Neurology 2005;65:1873-87

4. Harding B, Copp AJ. Malformations. In: Graham DI, Lantos PL, eds. Greenfield's Neuropathology. 6th ed. London: Arnold; 1997:397-533

5. Barkovich AJ, Lindan CE. Congenital cytomegalovirus infection of the brain: imaging analysis and embryologic considerations. AJNR Am J Neuroradiol 1994;15:703-15

6. Crome L, France NE. Microgyria and cytomegalic inclusion disease in infancy. J Clin Pathol 1959;12:427-34

7. Barkovich AJ, Rowley H, Bollen A. Correlation of prenatal events with the development of polymicrogyria. AJNR Am J Neuroradiol 1995; 16:822-27

8. Guerrini R, Parrini E. Neuronal migration disorders. Neurobiol Dis 2010;38:154-66

9. Robin NH, Taylor CJ, McDonald-McGinn DM, et al. Polymicrogyria 
and deletion 22q11.2 syndrome: window to the etiology of a common cortical malformation. Am J Med Genet A 2006;140:2416-25

10. Chang BS, Piao X, Bodell A, et al. Bilateral frontoparietal polymicrogyria: clinical and radiological features in $\mathbf{1 0}$ families with linkage to chromosome 16. Ann Neurol 2003;53:596-606

11. Chang BS, Piao X, Giannini C, et al. Bilateral generalized polymicrogyria (BGP): a distinct syndrome of cortical malformation. Neurology 2004;62:1722-28

12. Guerrini R, Dubeau F, Dulac O, et al. Bilateral parasagittal parietooccipital polymicrogyria and epilepsy. Ann Neurol 1997;41: 65-73

13. Guerrini R, Genton P, Bureau M, et al. Multilobar polymicrogyria, intractable drop attack seizures, and sleep-related electrical status epilepticus. Neurology 1998;51:504-12

14. Guerrini R, Barkovich AJ, Sztriha L, et al. Bilateral frontal polymicrogyria: a newly recognized brain malformation syndrome. Neurology 2000;54:909-13

15. Kuzniecky R, Andermann F, Guerrini R. Congenital bilateral perisylvian syndrome: study of 31 patients. Lancet 1993;341:608-12

16. Barkovich AJ. Morphologic characteristics of subcortical heterotopia: MR imaging study. AJNR Am J Neuroradiol 2000;21:290-95

17. Wieck G, Leventer RJ, Squier WM, et al. Periventricular nodular heterotopia with overlying polymicrogyria. Brain 2005;128: 2811-21

18. Galaburda AM, Sherman GF, Rosen GD, et al. Developmental dyslexia: four consecutive patients with cortical anomalies. Ann Neurol 1985;18:222-33

19. Guerrini R, Dobyns WB, Barkovich AJ. Abnormal development of the human cerebral cortex: genetics, functional consequences and treatment options. Trends Neurosci 2008;31:154-62

20. Barkovich AJ. Current concepts of polymicrogyria. Neuroradiology 2010;52:479-87

21. Cushion TD, Dobyns WB, Mullins JG, et al. Overlapping cortical malformations and mutations in TUBB2B and TUBA1A. Brain 2013; 136:536-48

22. Bahi-Buisson N, Poirier K, Boddaert N, et al. GPR56-related bilateral frontoparietal polymicrogyria: further evidence for an overlap with the cobblestone complex. Brain 2010;133:3194-209

23. Devisme L, Bouchet C, Gonzales M, et al. Cobblestone lissencephaly: neuropathological subtypes and correlations with genes of dystroglycanopathies. Brain 2012;135:469-82

24. Singer K, Luo R, Jeong SJ, et al. GPR56 and the developing cerebral cortex: cells, matrix, and neuronal migration. Mol Neurobiol 2013;47:186-96

25. Cho ZH, Kim YB, Han JY, et al. New brain atlas-mapping the human brain in vivo with 7.0 T MRI and comparison with postmortem histology: will these images change modern medicine? Int J Imag Syst Tech 2008;18:2-8

26. Abosch A, Yacoub E, Ugurbil K, et al. An assessment of current brain targets for deep brain stimulation surgery with susceptibilityweighted imaging at 7 Tesla. Neurosurgery 2010;67:1745-56

27. Metcalf $\mathrm{M}, \mathrm{Xu} \mathrm{D}$, Okuda DT, et al. High-resolution phased-array MRI of the human brain at 7 Tesla: initial experience in multiple sclerosis patients. J Neuroimaging 2010;20:141-47

28. van der Kolk AG, Hendrikse J, Zwanenburg JJ, et al. Clinical applications of 7 T MRI in the brain. Eur J Radiol 2013;82:708-18

29. Tallantyre EC, Dixon JE, Donaldson I, et al. Ultra-high-field imaging distinguishes MS lesions from asymptomatic white matter lesions. Neurology 2011;76:534-39

30. Conijn MM, Geerlings MI, Biessels GJ, et al. Cerebral microbleeds on MR imaging: comparison between 1.5 and 7T. AJNR Am J Neuroradiol 2011;32:1043-49

31. Madai VI, von Samson-Himmelstjerna FC, Bauer M, et al. Ultrahighfield MRI in human ischemic stroke: a 7 Tesla study. PloS One 2012;7:e37631

32. Mönninghoff C, Maderwald S, Wanke I. Pre-interventional assessment of a vertebrobasilar aneurysm with 7 Tesla time-of-flight MR angiography. Rofo 2009;181:266-68
33. Dammann P, Barth M, Zhu Y, et al. Susceptibility weighted magnetic resonance imaging of cerebral cavernous malformations: prospects, drawbacks, and first experience at ultra-high field strength (7-Tesla) magnetic resonance imaging. Neurosurg Focus 2010;29:E5

34. Lupo JM, Li Y, Hess CP, et al. Advances in ultra-high field MRI for the clinical management of patients with brain tumors. Curr Opin Neurol 2011;24:605-15

35. Cosottini M, Frosini D, Pesaresi I, et al. MR imaging of the substantia nigra at $7 \mathrm{~T}$ enables diagnosis of Parkinson disease. Radiology 2014;271:831-38

36. Kwon DH, Kim JM, Oh SH, et al. Seven-tesla magnetic resonance images of the substantia nigra in Parkinson disease. Ann Neurol 2012;71:267-77

37. Costagli M, Kelley DA, Symms MR, et al. Tissue border enhancement by inversion recovery MRI at 7.0 Tesla. Neuroradiology 2014;56:517-23

38. Leventer RJ, Jansen A, Pilz DT, et al. Clinical and imaging heterogeneity of polymicrogyria: a study of $\mathbf{3 2 8}$ patients. Brain 2010; 133:1415-27

39. Barkovich AJ. Abnormal vascular drainage in anomalies of neuronal migration. AJNR Am J Neuroradiol 1988;9:939-42

40. Guerrini R, Dravet C, Raybaud C, et al. Epilepsy and focal gyral anomalies detected by MRI: electroclinico-morphological correlations and follow-up. Dev Med Child Neurol 1992;34:706-18

41. Chassoux F, Landre E, Rodrigo S, et al. Intralesional recordings and epileptogenic zone in focal polymicrogyria. Epilepsia 2008;49:51-64

42. Guerrini R. Polymicrogyria and epilepsy. Epilepsia 2010;51:10-12

43. Maillard L, Koessler L, Colnat-Coulbois S, et al. Combined SEEG and source localisation study of temporal lobe schizencephaly and polymicrogyria. Clin Neurophysiol 2009;120:1628-36

44. Ramantani G, Koessler L, Colnat-Coulbois S, et al. Intracranial evaluation of the epileptogenic zone in regional infrasylvian polymicrogyria. Epilepsia 2013;54:296-304

45. Thompson JE, Castillo M, Thomas D, et al. Radiologic-pathologic correlation polymicrogyria. AJNR Am J Neuroradiol 1997;18:307-12

46. Friede RL. Developmental Neuropathology. Berlin: Springer-Verlag; 1989

47. Golden JA, Harding BN. Pathology and Genetics: Developmental Neuropathology. Basel: ISN Neuropath Press; 2004

48. Judkins AR, Martinez D, Ferreira P, et al. Polymicrogyria includes fusion of the molecular layer and decreased neuronal populations but normal cortical laminar organization. J Neuropathol Exp Neurol 2011;70:438-43

49. Marques Dias MJ, Harmant-van Rijckevorsel G, Landrieu P, et al. Prenatal cytomegalovirus disease and cerebral microgyria: evidence for perfusion failure, not disturbance of histogenesis, as the major cause of fetal cytomegalovirus encephalopathy. Neuropediatrics $1984 ; 15: 18-24$

50. Pryor J, Setton A, Berenstein A. Venous anomalies and associated lesions. Neurosurg Clin N Am 1999;10:519-25

51. Simonati A, Colamaria V, Bricolo A, et al. Microgyria associated with Sturge-Weber angiomatosis. Childs Nerv Syst 1994;10:392-95

52. Terdjman P, Aicardi J, Sainte-Rose C, et al. Neuroradiological findings in Sturge-Weber syndrome (SWS) and isolated pial angiomatosis. Neuropediatrics 1991;22:115-20

53. Cagneaux M, Paoli V, Blanchard G, et al. Pre- and postnatal imaging of early cerebral damage in Sturge-Weber syndrome. Pediatr Radiol 2013;43:1536-39

54. Comi AM. Pathophysiology of Sturge-Weber syndrome. J Child Neurol 2003;18:509-16

55. Dvorak K, Feit J, Juránková Z. Experimentally induced focal microgyria and status verrucosus deformis in rats: pathogenesis and interrelation-histological and autoradiographical study. Acta Neuropathol 1978;44:121-29

56. Aeby A, Guerrini R, David P, et al. Facial hemangioma and cerebral corticovascular dysplasia: a syndrome associated with epilepsy. Neurology 2003;60:1030-32

AJNR Am J Neuroradiol 36:309-16 Feb 2015 www.ajnr.org 
57. Evrard P, Kadhim H, de Saint-Georges P, et al. Abnormal development and destructive processes of the human brain during the second half of gestation. In: Evrard P, Minkowski A, eds. Developmental Neurobiology. New York: Vevey-Raven Press; 1989:21-41

58. Richman DP, Stewart RM, Caviness VS Jr. Cerebral microgyria in a 27-week fetus: an architectonic and topographic analysis. J Neuropathol Exp Neurol 1974;33:374-84

59. Suzuki M, Choi BH. Repair and reconstruction of the cortical plate following closed cryogenic injury to the neonatal rat cerebrum. Acta Neuropathol 1991;82:93-101

60. Abdollahi MR, Morrison E, Sirey T, et al. Mutation of the variant alpha-tubulin TUBA8 results in polymicrogyria with optic nerve hypoplasia. Am J Hum Genet 2009;85:737-44

61. Breuss M, Heng JI, Poirier K, et al. Mutations in the beta-tubulin gene TUBB5 cause microcephaly with structural brain abnormalities. Cell Rep 2012;2:1554-62

62. Guerrini R, Dobyns WB. Malformations of cortical development: clinical features and genetic causes. Lancet Neurol 2014;13: $710-26$

63. Jaglin XH, Chelly J. Tubulin-related cortical dysgeneses: microtubule dysfunction underlying neuronal migration defects. Trends Genet 2009;25:555-66

64. Poirier K, Saillour Y, Bahi-Buisson N, et al. Mutations in the neuronal ss-tubulin subunit TUBB3 result in malformation of cortical development and neuronal migration defects. Hum Mol Genet 2010;19:4462-73

65. Shain C, Ramgopal S, Fallil Z, et al. Polymicrogyria-associated epilepsy: a multicenter phenotypic study from the Epilepsy Phenome/Genome Project. Epilepsia 2013;54:1368-75

66. Im K, Pienaar R, Paldino MJ, et al. Quantification and discrimination of abnormal sulcal patterns in polymicrogyria. Cereb Cortex 2013;23:3007-15

67. Guerrini R, Filippi T. Neuronal migration disorders, genetics, and epileptogenesis. J Child Neurol 2005;20:287-99 